\title{
DOMAIN STUDIES OF COCr WITH PERPENDICULAR ANISOTROPY
}

\author{
J.C. Iodder, D. Wind, Th.J.A. Popma, A. Hubert*
}

University of Twente, P.O. Box 217, 7500 AE Enschede, The Netherlands. *University of Erlangen-Nurnberg, Martensstrasse 7, D-852 Erlangen, FRG.

Albstract. R.F. Magnetron sputtered CoCr films (79/21 ats) with various thicknesses are magnetically characterized. The domain structure is observed by digitally enhanced Kerr microscopy and depends on the Hc/Hk values of the samples. For low and high coercivity films a cormparison is made between the measured vSM hysteresis, domain period and a theoretical domain model. The domain shape is a function of the magnetic history of the sample and the bending created by the deposition process.

\section{INTRODUCTION}

The reversal mechanism of magnetization in sputtered $\mathrm{CoCr}$ layers for perpendicular recording has still not been completely clarified. Studying the domain configuration can contribute to the solution of this problem.

The magnetic properties of $\mathrm{CoCr}$ layers are strongly influenced by variations in microstructure, morphology and chemical homogeneity. Initial layer effects, $\mathrm{Cr}$ segregations at the colum boundaries and surface are very important for the final behaviour of the domains. The morphology also depends on the sputtering method and parameters used (mainly substrate temperature and Ar gas pressure). From the perpendicular (e.a.) hysteresis $100 \mathrm{p}$, measured with the VSM, we have in some cases, observed a typical shoulder which indicated bubble-stripe damains. In order to understand more about the micromagnetics of such layers one has to correlate the morphology, nagnetic parameters and domains. Lorentz transmission electron microscopy and digitally enhanced Kerr microscopy have been used for danain observations in COCr layers. A $1 \mathrm{MeV}$ TEM has to be used for observing $\operatorname{coc} x$ layers thicker than $\varnothing .1 \mu \mathrm{m}$, up to a maximum of $ø .3 \mu \mathrm{m}[1,2]$. A specially designed sample stage to exclude a perpendicular lens field from the specimen during domain observations is essential $[1,3]$. Different domain configurations with in-plane magnetization are observed in thin (<60 nm) CoCr layers [2-4]. This can be explained by variations in the preparation methods and consequently differences in morphology, structure and chemical homogeneity. None of the samples used has a well oriented hop-axis. For a good textured layer (co77cr23), having a thickness of $50 \mathrm{~nm}$, stripe domains with a width of $\emptyset .1 \mu \mathrm{m}$ were observed [2]. A maze domain pattern, known from bubble-like materials, is observed in films of $\vartheta .1 \mu \mathrm{m}$ thickness having a composition of Co82Crl8 [5]. Very thin layers $(30 \mathrm{~nm})$ with in-plane magnetization show the so-called ripple structure in Co89CrlI [4] and Co79Cr21 [5]. Different damain forms were observed in films with much better orientation and increased thickness $(>\varnothing .1 \mu \mathrm{m})[2-5]$.

A magnetic black-and-white dot structure with a distance between the dots of the same order as the columnar diameter has been shown by [3]. In this case the film morphology (one colum consists of several crystallites) is totally different from the equiaxed crystal structure present in [2] which shows a stripe domain width in the order of the crystal size. Cocr films in the range between $17-3 \varnothing$ ats $\mathrm{Cr}$ with a $\mathrm{Cr}$ segregation into the column boundaries show dot-like Lorentz images which have the same size as the colum diameter [5]. Stripe domain widths $(<\emptyset .1 \mu \mathrm{m})$ having the same size as the column-diameter are also observed in [1]. It was also shown that from relatively thick films $(\emptyset .3 \mu \mathrm{m})$, which are more realistic for recording applications, the domain width is the same as the colum size [2] and it is suggested that the domain structure consists of a chain of crystals. Based on neutron depolarization experiments and M.O. measurements of the surface of our $\mathrm{CoCr}$ films it can be concluded that the damain width is proportional to $h^{x}$, where $x$ is about $\emptyset .8$ for RF magnetron sputtered films [6-1ø]. This value is closer to the calculated power of $2 / 3$ for a branched domain structure [II] than $1 / 2$ predicted for the Kittel configuration [12]. It can also be concluded that reversed spike domains can exist and will influence the reversal mode above the critical thickness of magnetron sputtered coCr layers $(500-800 \mathrm{~nm})$ [10]. Unfortunately (spike-) donains cannot be really visualized by neutron depolarization.

\section{EXPERIMFNTAL PROCEIUURE}

The Cocr layers are deposited on Si-substrates by RF magnetron sputtering, having a $3^{\prime \prime}$ target, back pressure $1.5 \times 10-7$ mbar and $\operatorname{Par}=6 \times 10^{-3}$ mbar. The film cormosition is around $2 \mathrm{l}$ ato $\mathrm{Cr}$ and the thickness $(\mathrm{h})$ is determined by XRF. Magnetic measurements are carried out by VSM and torque magnetometer. Substrate material is $\mathrm{Si}$ (1øø) single crystal $1 \varnothing \times 1 \varnothing \mathrm{mm}^{2}$. Damains are observed by a digitally enhanced Kerr microscope. The layers are covered with an evaporated $1 / 4 \lambda$ zns layer. Roughness and bending of the deposited substrate can be measured with the Sloan Dektak $3 \emptyset 3 \emptyset$ Stylus instrument. Column diameter and morphology are detemined by STEM observation using a Jeol $200 \mathrm{cx}$ microsoope. The relevant sample properties are given in Table $I$.

\begin{tabular}{|c|c|c|c|c|c|c|c|c|}
\hline Sample & $\begin{array}{c}\mathrm{Ms} \\
\mathrm{kA} / \mathrm{m}\end{array}$ & $\begin{array}{l}\mathrm{h} \\
\mathrm{nm}\end{array}$ & $\begin{array}{c}\mathrm{HC} \\
\mathrm{kA} / \mathrm{m}\end{array}$ & $\frac{\mathrm{Kl}}{\mathrm{J} / \mathrm{m}^{3 *} 1 \delta^{4}}$ & \begin{tabular}{|c|}
$\mathrm{Hk}$ \\
$\mathrm{kA} / \mathrm{m}$
\end{tabular} & $\begin{array}{c}\mathrm{Hc} / \mathrm{FkK} \\
8 \\
8\end{array}$ & $\begin{array}{l}\text { Pmr } \\
\mathrm{nm}\end{array}$ & $\begin{array}{l}\text { damain } \\
\text { shape }\end{array}$ \\
\hline 1 & 462 & 520 & 8 & 13.2 & 525 & 1.5 & 660 & long \\
\hline 2 & 410 & 1230 & 8 & 14.3 & 555 & 1.4 & 910 & stripes \\
\hline 3 & 441 & 760 & 10 & 16.0 & 575 & 1.7 & 790 & \\
\hline$\overline{4}$ & 423 & 700 & 18 & 14.8 & 555 & 3.2 & 730 & short \\
\hline 5 & 415 & 1300 & 24 & & 595 & 4.0 & 960 & stripes \\
\hline 6 & 413 & 1220 & 46 & 16.8 & 615 & 7.5 & 850 & dot- \\
\hline 7 & 434 & 7000 & 60 & 18.4 & 675 & 8.9 & 840 & like \\
\hline 8 & 426 & 1436 & 40 & 16.4 & 615 & 6.5 & 820 & \\
\hline
\end{tabular}

\section{M.O. KERR OBSERVATIONS}

The first Kerr observations on $\operatorname{cocr}$ layers with divergent properties are published in [13]. There a specially designed digitally enhanced Kerr microscope with an image processing system is used. The films used have a composition from 17 to $2 \mathrm{l}$ at: $\mathrm{Cr}$, thicknesses from 96-622 $\mathrm{mm}$ and coercivities from $1 \varnothing-$ $60 \mathrm{kA} / \mathrm{m}$ with one sample of $90 \mathrm{kA} / \mathrm{m}$. They all show a more or less stripe-domain structure at remanence. It was concluded from this that all samples reversed by domain-wall movenent as a continuous medium. Using the same microscope mentioned above, we came to the conclusion with our preliminary results [14] that we have to divide our cocr layers into 3 categories namely: low Hc (long stripe), medium Hc with short stripes $(2 \emptyset<\mathrm{Hc}<4 \emptyset \mathrm{kA} / \mathrm{m})$ and high coercivity films with a dot-like domain structure. The domain behaviour is clearly connected with the shoulder in the perpendicular hysteresis loop. The Kerr observation method has its limitations in the resolution $(\sim 260 \mathrm{~nm})$ and depth of information $(\sim 15 \mathrm{~mm})$. An advantage in relation to transmission Lorentz microscopy is that (surface) magnetic information can be obtained fram all thicknesses without any thinning techniques with their consequent disadvantages. A comparison between both observation methods is mentioned in [5], i.e. the Kerr image of an $\varnothing .6 \mu \mathrm{m}$ thick sample (2ø at $\mathrm{Cr}$ ) and a Lorentz image of $6.1 \mu \mathrm{m}$ thick (18 ato $\mathrm{Cr}$ ) both showing a stripe-like domain pattern. The interpretation of these images is not so simple. The differences in thickness, chemical cormosition and eventually removal from the original substrate will have an important influence on the domain behaviour. Besides, observation of double layered samples is cormpletely impossible with the Lorentz method. Another advántage of Kerr microscopy is the possibility of applying fields up to $800 \mathrm{kA} / \mathrm{m}$. For most of the $\mathrm{CoCr}$ layers the domain process through the whole hysteresis loop can be observed $[13,14]$ within the limitation of the resolving power. 


\section{EXPERIMENTAL RESULTS}

A series of samples was made and the measured and calculated results from Kerr observations, VSM and torque magnetoneter are given in table $I$. The $Q$-factor of our samples is derived from $Q=K 1 / \emptyset .5 \mu_{0} \mathrm{Ms}^{2}$. For $Q>1$ the perpendicular axis will thus be the direction of preferred magnetization in the case of a uniform magnetized layer. In the demagnetized state the magnetization is split up into donains. The condition for stable perpendicular ragnetization $\mathrm{KI}>\mathrm{EC}$ is expressed in the Qfactor. for our samples Q varies from 1.30-1.50. The domain shape is not dependent on this Q-range, but on the coercivity of the layers. Of course, the $\mathrm{HC}$ is strongly dependent on the reversal mechanism and the magnetic morphology ( $\mathrm{Cr}$ segregation at the column boundaries). In order to explain the observed stripe domains we have used the Kooy and Enz nodel [15]. The two relevant equations which have to be solved are explicitely given in [4].

The parameters used are: film thickness (h), material parameter $(\lambda)$, domain period (P) and $\mu=1+M s / 2 \mathrm{Kl}$ which was kept constant at 1.70 .

Both the equations are solved for several pairs of $h / \lambda$ and $\mathrm{H} / \mathrm{Ms}$ values. In Fig. 1 the relation $\mathrm{M} / \mathrm{Ms}$ vs. $\mathrm{H} / \mathrm{Ms}$ and in $\mathrm{Fig} .2$ $\mathrm{P} / \lambda$ vs. $\mathrm{H} / \mathrm{Ms}$ are shown. In both plots (see broken lines) $\mathrm{h} / \lambda$ is used as a parameter. From VSM measurements we know the hysteresis loop and from this we detemine the slope $\mathrm{T}=\mathrm{dM} / \mathrm{dH}$ for $M=\emptyset$ or $H=+H C$. This initial slope is the most relevant parameter. An other relevant parameter $\mathrm{D}$ is measured from the Kerr photographs as a function of the applied field.

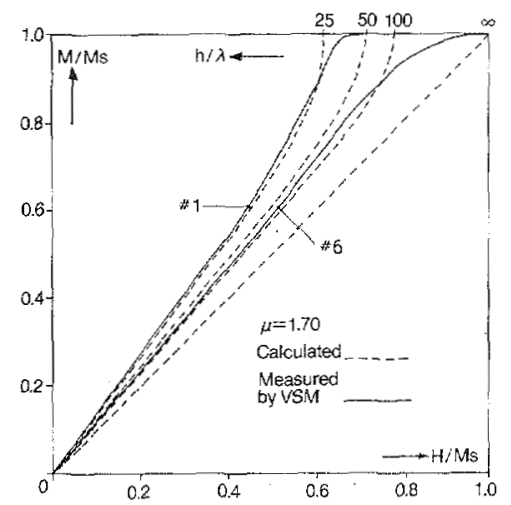

Fig. $1 \mathrm{M} / \mathrm{Ms}$ vs. $\mathrm{H} / \mathrm{Ms}$ from measured and calculated (with $\mathrm{h} / \lambda$ as a parameter) hysteresis for low (\#1) and high (\#6) coercivity.

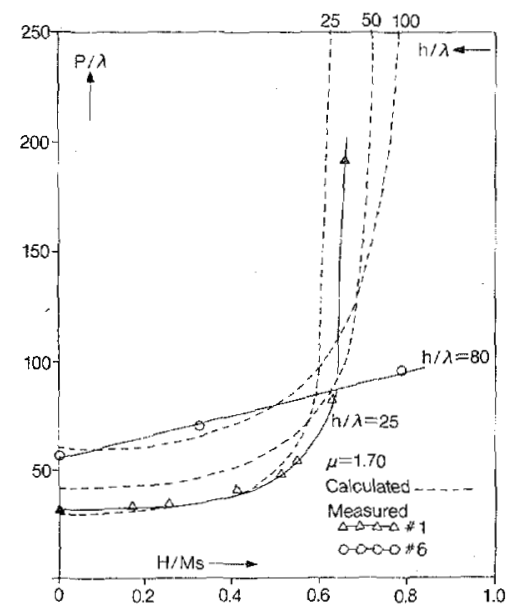

Fig. 2 P/ $\lambda$ vs. $H_{j}: i s$ (see caption Fig. 1 ).

Both $\mathrm{P}$ and $\mathrm{T}$ are detennined independently and must agree simultaneously with the theoretical calculation for a certain $h / \lambda$ in both normalized figures 1 and 2. For films with a low HC (\#1) and a high HC (\#6) the M/Ms vs. H/Ms values calculated (dotted line) and measured (solid line) are given in Fig. 1.
The measured slope is also in good agreenent with the calculated line for $h / \lambda=25 \mathrm{in} \mathrm{Fig.} \mathrm{2.} \mathrm{For} \mathrm{this} \mathrm{sarmple} \lambda=20.8 \mathrm{~nm}$, $\sigma_{\mathrm{W}}=\mu_{\mathrm{O}} \mathrm{M}_{\mathrm{S}} 2 \lambda=4.2^{*} 10^{-3} \mathrm{~J} / \mathrm{m}^{2}, \mathrm{~A}=(1 / \mathrm{kl}) .\left(\sigma_{\mathrm{w}} / 4\right)^{2}=$ $8.4 \times 1 \varnothing-12 \mathrm{~J} / \mathrm{m}$ and $1=\pi \sqrt{\mathrm{A}} / \mathrm{kl}=25 \mathrm{~nm}$.

Another low Hc filru (\#2) has also been measured and calculated. Here we found $h / \lambda=55$ as the best fit. For sample \#6 (high Ho) we could not fitt both curves (see Figs. 1,2).

We have also compared the size and shape of the domain period of \#2 in the remanent state after saturation with perpendicular DC and demagnetizing fields (Fig. 3).

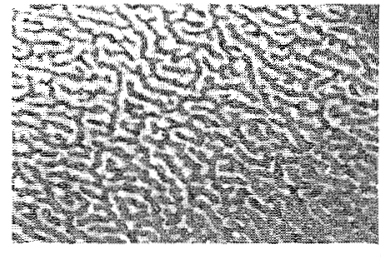

$5 \mu$

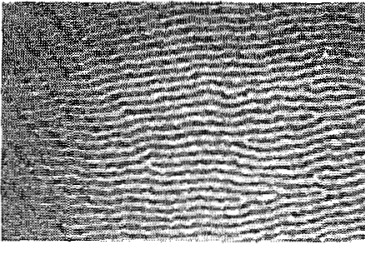

Fig. 3 Donlain configurations of a low coercivity film in remanent (1.eft) and demagnetized (right) state.

At remanent state $P M r=910 \mathrm{~nm}$ and after demagnetization Fo $=600 \mathrm{~mm}$. This minimm energy position of the stripes results in a very good parallel orientation. Damain doservations in the field free state show the magnetic history of the sample. Also different danain configurations can be observed after partial saturation and applying a field under an angle [13]. For a high Ho sample (\#6) the dot-like domain shape at denagnetizing field can be roughly given as $P O=665 \mathrm{~nm}$. Typical shoulders in VSM and M.O. Kerr hysteresis loops are not seen in the high Ho films. The calculated model by means of $P$ and $T$ is not in good agreenent for such films (see Figs. 1,2). We have to realize that the method we used makes the following assumptions namely: the domainwall energy is independent of $\mathrm{h}$. the wall volune is neglected and, last but not least, the coercivity is zero.

Stresses have a lot of influence on the domain configuration. The bending of the sarples is measured: A line scan over the total sample length in two orthogonal directions is made. The curvature (e.g. the strain) is not always isotropic over a certain area and can vary from place to place in the same sample. We found that samples have a different bending in the orthogonal directions showing straight stripe domains (see Fig. 4a). More or less meandered domains are seen in Fig. 4b which is made from a position with equal bending in two directions.
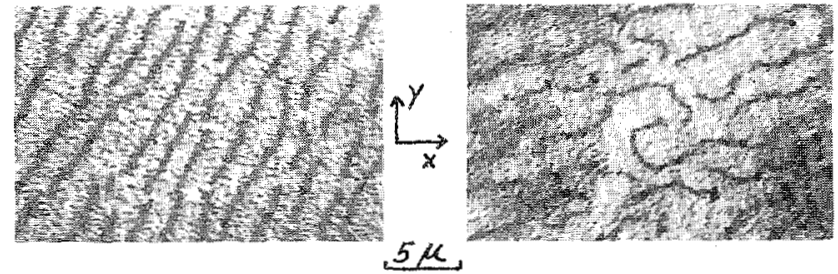

a) asymmetrical $(x>y)$

b) symmetrical ( $x=y)$

Fig. 4 Domains of $\$ 2$ at $\mathrm{Ha}=260 \mathrm{kA} / \mathrm{m}$ as a function of the bending in two orthogonal directions (xy).

At the moment we have the impression that stress in our magnetron sputtered films is higher than in our RF sputtered anes [4]. Consequently the stress will have to be studied in more detail in relation to the total anisotropy factor in the filns.

\section{MORPHOLOGY}

Cross-sections were studied with the SiFM mode of the electron microscope. The fractional surfaces were prepared by cooling the samples in liquid $\mathrm{N}_{2}$ before breaking them, to obtain a brittle fracture. The columar-like morpholgy can be clearly seen in all our films independent of the Hc/Hk value. 
For samples \#3, 5 and 8 respectively an average column size D (measured at the surface) of $4 \varnothing, 45$ and $7 \varnothing \mathrm{mm}$ is determined. Consequently Pme/D values are 20, 21 and 12. From another low (\#2) and high (\#6) coercivity film we have measured po (see ahove) and using these values we came to the conclusion that one domain consists of 4-8 columns, for these films.

We also have to realize that the column size is a function of the layer thickness. Studying the fractional surfaces it can be seen that the three films show a typical fractured plane close to the substrate. This is interpreted as belonging to the initial layer which is also seen in the in-plane VSM graphs of the same samples.

\section{COERCIVITY}

The coercivity in ferromagnetic layers with perpendicular anisotropy is detemined by two factors.

1. The type of magnetization process involved i.e. is the magnetization reversed by (coherent) rotation or by domain-wall movement?

2. Morphology of the layer i.e. shape and dimensions of the crystallites (columis), nature of the boundaries, surface and initial layer properties.

In fact these two factors are strongly interrelated and their properties are strongly influenced by the deposition parameters such as termperature, Par etc.. Based on general knowledge about ferromagnetic films we can conclude that the presence of stripe domains in CoCr must be quite conmon because the layer splits up into such a configuration by reducing its demagnetizing energy. Theoretical support for reversed domains in uniaxial thin layers with perpendicular anisotropy is provided by $[11,16]$. From research on permanent magnets, supporting arguments for the relation coercivity and reversal mechanism are given by [17]. In polycrystalline and/or columar-like materials the critical damain size is difficult to estimate because it is influenced by the presence of neighbouring particles and their interactions. If the magnetization reversal takes place by coherent rotation ilc must be equal to the. Hk. In our case all the layers have Hc<Hk. An explanation for this effect can be made by introducing the so-called fanning, curling and buckling modes. These can in principle, only occur in layers in which the magnetic anisotropy is mainly produced by shape anisotropy. In $\mathrm{CoCr}$ the main anisotropy is configurated by magneto crystalline anisotropy. On the other hand experimental work on high coercivity Co or Co-P layers [18-2ø] shows domain structures independent of the source of the magnetic anisotropy. The presence of a domain structure and the results of 'other measurements like hysteresis loss and HC vs. angle are not completely in line. This could perhaps be explained by the phenomena of interaction domains which were first introduced by [21]. Here particle exchange forces are negligible and the magnetic manents are mutually aligned by magnetostatic interactions [22]. Recently this model was mentioned as a possible mechanism in Cocr layers [23]. For low Hc the nucleation density is much lower than for high Hic ones. It is clearly shown that nuclei stripe out at the tips of the domain after decreasing the applied field [13,14]. The coservable size of the dots (coming from saturation) in high Ho filns is determined by the resolving power of the Kerr microscope. Nevertheless these dots do not expand very much due to the very close surrounding of neighbouring dots. The reversal in one column could be, depending on its dimensions, initiated by a reversed domain. These small sizes cannot be resolved by Kerr microscopy and it can be accepted that such domains are no longer present in thinned TFM specimens. The particle behaviour without exchange coupling is suggested by $\mathrm{Cr}$ segregation at the colum boundary. At the moment only prelimary resulsts are available from Scanning Auger Microspectroscopy (SAM) and wet chenical etch procedures. From the latter we found only qualitatively a higher $\mathrm{Cr}$ content at the surface than in the bulk of all our samples. From a SAM measurement of a fractional surface of a high coercivity film we came the the conclusion that there is a Cr segregation at the column boundaries.

\section{CONCLUSIONS}

Different domain configurations can be expected as a function of the coercivity. Factors like stress and magnetic history play an important role. For low He material the stripe domain theory is in good agreement with the measured $P$ values from Kerr observations and the hysteresis loop.

one remanent domain (dot, stripe) of all our films consists of several columas.

A direct correlation between the type of domains and same aspects of the macroscopical hysteresis loop (IIc, shoulder) can be made (see also $[14,24]$ ).

Stress plays an important role in the damain behaviour.

\section{REFERENCES}

[1] K. Ouchi and S. Iwasaki, IFEE Trans. Magn. MAG-18, 111ø, (1982).

[2] P.J. Grundy and M. Ali, J. Magn. Magn, Mat., 40, 154 (1983).

[3] M. Ohkoshi and T. Kusuda, Japan. J. Appl. Phys. 22, L]30, (1983).

[4] T. Wielinga, Thesis, University of Twente, Enschede, The Netherlands, (1983).

[5] H. Hoffmann, IEEE Trans. Magn. MAG-22, 472, (1986).

[6] K. Henmes, J.C. Lodder, M.Th. Rekveldt and W.H. Kraan, J. Phys. D: AppI. Phys. 17, L157, (1984).

[7] W.H. Kraan, M.Th. Rekveldt, K. Henmes and J.C. Lodder, Fhysica 136B, 446, (1986).

[8] W.H. Kraan, M.Th. Rekveldt, K. Hermes and J.C. Lodder, IEEE Trans. Magn. MAG-23, 65, (1987).

[9] K. Hemmes, W. Lisowski, J.C. Lodder, L.J. Hanekarm and Th.J.A. Popma, J. Phys. D: Appol. Phys. 19, 1311, (1986).

[1ø] K. Hermes, J.C. Lodder and Th.J.A. Popma, IEEE Trans. Magn. MAG-23, 150, (1987).

[11] A. Hubert, IEFE Trans. Magn. MAG-21, 16ø4, (1985).

[12] C. Kittel, Phys. Rev. 76, 965, (1946).

[13] F. Schmidt and A. Hubert, J. Magn. Magn. Mat., 61, 307, (1986).

[14] J.C. Lodder, D. Wind, G.E. v. Dorssen, Th.J.A. Popna and A. Hubert, IFEFE Trans. Magn. MAG-23, 214, (1987).

[15] C. Kooy and U. Enz, Philips Res. Repts. 15, 7, (1960).

[16] I. Privorotskii, Thermodymamic Theory of Domain Structures, J. Wiley \& Sons, New York, (1976).

[17] J.D. Livingston, J. App1. Phys. 52, 2544, (1981).

[18] G.A. Jones and F. Farnsworth, Phys. Stat. Sol. 79, 970, (1962).

[19] J. Daval and D, Randet, IEEE Trans. Magn. MAG-6, 768, (1976).

[20] T. Tanaka, K. Yazawa, H. Masuya, IEEE Trans. Magn. MAG-2l, 2896, (1985).

[21] D.J. Craik and E.D. Isaac, Proc. Phys. Soc. 76, 160, (1968).

[22] L.F. Bates, D.J. Craik and E.D. Isaac, Proc. Phys. Soc 79, 97ø, (1962).

[23] E.P. Wohlfarth, MRM Conference, Italy, (1986).

[24] Li. Cheng-Zhang and J.C. Lodder, Paper BA-ø8, Intermag '87. 